University of Nebraska - Lincoln

DigitalCommons@University of Nebraska - Lincoln

Eileen Hebets Publications

Papers in the Biological Sciences

2011

\title{
Chemical Communication in a Multimodal Context
}

Eileen A. Hebets

University of Nebraska - Lincoln, ehebets2@unl.edu

Aaron S. Rundus

University of Nebraska-Lincoln, arundus2@unl.edu

Follow this and additional works at: https://digitalcommons.unl.edu/bioscihebets

Part of the Behavior and Ethology Commons

Hebets, Eileen A. and Rundus, Aaron S., "Chemical Communication in a Multimodal Context" (2011).

Eileen Hebets Publications. 41.

https://digitalcommons.unl.edu/bioscihebets/41

This Article is brought to you for free and open access by the Papers in the Biological Sciences at DigitalCommons@University of Nebraska - Lincoln. It has been accepted for inclusion in Eileen Hebets Publications by an authorized administrator of DigitalCommons@University of Nebraska - Lincoln. 


\title{
Chemical Communication in a Multimodal Context
}

\author{
Eileen A. Hebets and Aaron Rundus \\ School of Biological Sciences, University of Nebraska-Lincoln, \\ Lincoln, NE 68588-0118, USA \\ Corresponding author - E. A. Hebets, email ehebets2@unl.edu
}

\begin{abstract}
All animals are equipped with multiple sensory systems (e.g., visual, chemical, acoustic, tactile, electrical, thermal), and signals perceived via these sensory systems facilitate communication. Such communication often involves displays that incorporate more than one signal from more than one sensory modality, resulting in multimodal signaling. The number of empirical and theoretical studies addressing issues of multimodal signaling is ever-increasing and this chapter highlights why crustaceans, as a taxonomic group, are ideal for advancing such studies. Early classifications of multimodal signaling sought to categorize signal components as either redundant or nonredundant, while more recent classifications lay out specific hypotheses relating to multimodal signal function. Two common empirical approaches used in studying multimodal signaling involve signal isolation and signal playback designs - both of which are extremely amenable to crustaceans.

Chemical communication is considered the oldest and most widespread channel for communication, and as such, it is not surprising that numerous crustaceans incorporate chemical signals into multimodal displays. In this chapter, we review multimodal signaling in crustaceans with a focus on those displays that incorporate a chemical component. Specifically, we highlight examples of taxa that combine chemical and hydrodynamic as well as chemical and visual cues. We conclude that despite the plethora of excellent studies examining crustacean responses to isolated signal components, relatively few studies are couched in a communication framework - ultimately limiting the conclusions that can currently be drawn with respect to multimodal signal evolution and function in crustaceans. We suggest that future studies using a hypothesis-testing framework of multimodal signal function could greatly advance our understanding of multimodal signaling in this group. Furthermore, studies involving signal manipulations and correlations between signaler attributes and variation in signal form could be extremely informative. These avenues are wide open for crustacean biologists. We argue that several aspects of crustacean
\end{abstract}


biology (e.g., their abundance, the ease with which they can be manipulated, the ease with which their environment can be manipulated, their morphological diversity, the diversity of habitats in which they live, etc.) make them ideal for studying multimodal signaling.

\section{Introduction}

All animals are equipped with multiple sensory systems (e.g., visual, chemical, acoustic, tactile, electrical, thermal) and signals perceived via these sensory systems facilitate communication. It has long been the goal of scientists to understand the selection pressures that influence signal form and function. Yet, despite the significant progress that has been made, it is becoming increasingly clear that animal displays are often complex, incorporating more than one signal or component, frequently from more than one sensory modality (i.e., multimodal Rowe 1999) into a single display. This recently acknowledged complexity of animal displays has increased the difficulty for conducting empirical studies of signal function, yet it has simultaneously given birth to exciting new theory.

Multimodal signals comprise a fascinating and widespread category of complex signaling in which components from more than one sensory modality are combined into a single multimodal display. The addition of signaling modalities presumably carries with it several costs such as increasing a signaler's energy expenditure, increasing its conspicuousness, and/or adding new avenues available for eavesdropping or aggressive mimicry (Hebets and Papaj 2005; Partan and Marler 2005). Why then is multimodal signaling so common? This question can be asked in several ways. For example, from an evolutionary viewpoint, we might ask whether general patterns regarding the way in which sensory modalities are combined can shed light on selection pressures driving the evolution of multimodal signaling. From an ecological point of view, we might ask whether ecological constraints can select for multimodal signaling. If the signaling environment is variable, and signal efficacy is tightly tied to environmental characteristics, simultaneously signaling in multiple modalities might prove advantageous. From a functional point of view, we might ask whether signals in different modalities, or in different combinations of modalities, are better at effectively eliciting the desired behavioral response(s) from signal targets. This latter line of questioning can then lead to more proximate examinations of how an animal's neural architecture influences the reception and processing of multisensory input. For those of us interested in integrative approaches to understanding trait evolution, multimodal signaling provides an excellent opportunity to simultaneously explore the evolution and function of a trait at multiple levels of analysis.

Although certainly not at the forefront of our minds, when pressed, most of us can relate well to the importance of multimodal signaling. Being part Sicilian for example, I (EAH) find that I rely heavily on gestures and body language when telling a story, teaching a class, etc. I typically incorporate gestures to em- 
phasize a point or to more clearly convey my verbal information. As a result, I am much more effective at conveying information in person, for example, than over the phone. Similarly, my now $31 / 2$-year-old daughter became proficient in multimodal communication while learning verbal language. My husband, Jay Storz, and I taught Jessie sign language well before she could speak. She was quick to pick up the signs she was taught and soon realized that she could further increase the efficacy of her communication by incorporating additional visual signals in the form of facial expressions - resulting in multicomponent visual signaling. Once her verbal vocabulary started to surface, Jessie began combining her visual signals with her verbal words and her language became truly multimodal. Depending upon both the situation and the information she wished to convey, Jessie's multimodal signaling (visual and acoustic) functioned to convey the same information (for example, because I could not figure out what she was trying to tell me using only one component, e.g., redundant signal hypothesis) or to convey multiple bits of information (e.g., multiple messages). Regardless of reason, as a receiver, I have no doubt that I was more likely to understand what my daughter was trying to tell me and to respond appropriately if I could both see and hear her.

As is often the case with new trends in research topics, much of the functional work on multimodal signaling has thus far focused on a few taxonomic groups (e.g., birds, spiders). Throughout this chapter, we emphasize why crustaceans should not be overlooked for their potential contribution to this field. We will call attention to several aspects of crustacean biology that make them excellent candidates for research on multimodal signaling, many of which are unique to the group (Figure 1). Furthermore, crustaceans rely heavily on chemical communication, a sensory modality deserving more attention in multimodal signaling. However, before delving deeper into crustacean multimodal communication, we will first lay the conceptual groundwork by describing the framework and general approaches used, as well as by providing a few well-studied examples from other taxonomic groups.

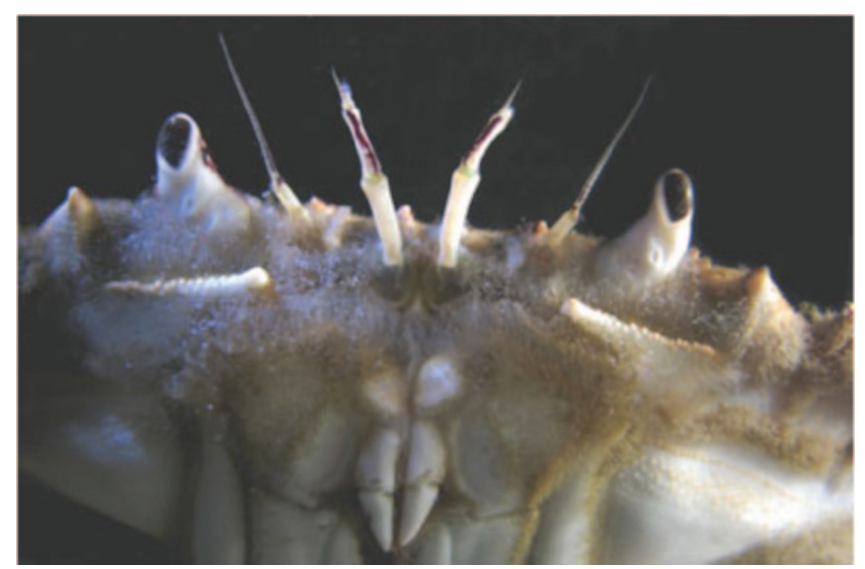

Figure 1. Head region of Ovalipes trimaculatus showing the antennae (which perceive chemical and hydrodynamic stimuli) and the stalked eyes (which perceive visual stimuli). Photograph by Iván A. Hinojosa. 


\section{General Aspects of Multimodal Signaling}

\subsection{Conceptual Framework of Multimodal Signaling}

The study of multimodal signaling is rich with theory, as evidenced by the plethora of recent reviews of the subject (Candolin 2003; Hebets and Papaj 2005; Partan and Marler 2005). Early classifications sought to place multimodal signals into categories of redundant versus nonredundant signals based upon receiver responses (Partan and Marler 1999), while more recent classifications attempt to lay out more specific hypotheses relating to complex signal function (Candolin 2003; Hebets and Papaj 2005). Much of the theoretic framework addresses the ultimate question - "why" - why use multimodal signals? This question is most commonly addressed from a content-based approach, with studies focusing on the characteristics of signaling individuals and exploring how these characteristics are, or are not, reflected in the form of their signal. In other words, contentbased approaches focus upon the purpose of the signal and the putative information it conveys. Using this approach, the "multiple messages" and "redundant," or "back-up," signals hypotheses are the most commonly tested functions of multimodal signaling (Møller and Pomiankowski 1993; Johnstone 1996). The "multiple messages" hypotheses simply propose that different signals reflect different information or content (Møller and Pomiankowski 1993; Johnstone 1996). For example, one modality may correlate with foraging history and another with parasite resistance. In contrast, the "redundant signal" hypothesis suggests that multiple signals provide the same information, with some error, and thus enable receivers to more accurately assess signal content (Møller and Pomiankowski 1993; Johnstone 1996). Several studies across numerous taxonomic groups have provided evidence for a multiple messages function of complex signaling, while fewer studies have found support for a role of backup, or redundant, signals (see Candolin 2003; Hebets and Papaj 2005).

Regardless of the information content of a signal, it is only effective if it can be produced, transmitted, perceived, and processed effectively. As such, signals are subject to what is referred to as efficacy-based selection in addition to content-based selection (Guilford and Dawkins 1991). An example of how efficacybased selection can drive the evolution of multimodal signaling is well-articulated by Candolin (2003) in her "multiple sensory environments" hypothesis of multiple cues used in mate choice. Stated simply, the transmission and detection of signals and cues is influenced by environmental conditions and thus variability in the signaling environment may select for multimodal signals independent of information content (see Candolin 2003; Hebets and Papaj 2005). In addition to environmental variability, there likely exists variability among receivers in terms of signal detection and processing, which may also exert selection pressure on signal form. Additionally, signals need not be independent and may interact such that one alters either the production, reception, or processing of another (Intersignal interaction hypotheses - Hebets and Papaj 2005), making some multimodal signals functional units upon which selection can act. In summary, 
there is a wealth of ideas regarding how multimodal signals function and why they might exist and many of these hypotheses remain untested - making multimodal signaling an exciting and timely focus of research (see Candolin 2003; Hebets and Papaj 2005).

While progress has been made regarding multimodal signal function within select taxonomic groups, there are countless questions that remain unanswered. For instance, published examples of multimodal signaling most often include displays typified by the simultaneous production and transmission of signals and/or components from more than one sensory modality (see Candolin 2003; Hebets and Papaj 2005), yet it is likely that some (even many) multimodal signals function in a sequential fashion. The importance of simultaneous versus sequential production, transmission, and perception of multimodal signals is a relatively untouched topic in theoretic discussions of multimodal signal function and exemplifies the work that remains to be done in this field. Interestingly, the sequential arrival of information to receivers has previously received attention from crustacean biologists (e.g., Hazlett and McLay 2000), setting a precedent for similar future studies.

\subsection{Approaches to Studying Multimodal Signaling}

Depending upon the questions of interest, approaches to studying multimodal signaling may include morphological studies, ecological studies, behavioral manipulations and assays, psychological experiments, histology, electrophysiology, phylogenetic analyses, and computational studies. Generally, however, studies of multimodal signaling fall within the fields of behavior and/or neurobiology. The "how" questions - how is simultaneous input from multiple sensory systems received and potentially integrated in the nervous system-are commonly addressed with neurobiological techniques such as histology and electrophysiology. In contrast, the "why" questions - why do animals use multiple modalities when communicating - are addressed with a whole suite of complementary behavioral techniques and are rife with theoretical hypotheses (see above).

An approach championed by Partan and Marler $(1999,2005)$ to address the "why" questions of multimodal signaling involves comparing receiver responses to signal components both in isolation and jointly in an attempt to categorize the components as redundant versus nonredundant. In many taxonomic groups, this presents a difficult, frequently insurmountable challenge, yet as we will demonstrate subsequently, crustacean researchers can readily overcome this challenge. Currently, the two most common ways to assess receiver responses are using (1) signal isolation and/or (2) signal playback experiments (for discussion of approaches see Uetz and Roberts 2002; Hebets 2008). Signal isolation experiments involve manipulating signal production, signal transmission, or receiver reception such that a receiver's response to a single signal in isolation can be assessed. Such experiments require animals for which such manipulations are feasible-such as crustaceans. Signal playback experiments rely on technology such as video/acoustic playback and/or robotics, again, requiring taxonomic groups 
amenable to such techniques - again, such as crustaceans. Below, we will use our own study systems to highlight two noncrustaceans examples of studies utilizing multiple techniques to address questions of multimodal signal evolution and function.

\subsection{Example I: A Comparative Approach to Multimodal Signal Function in Spiders}

Male Schizocosa wolf spiders exhibit tremendous variation in courtship displays with respect to their use of visual and seismic (substratum-coupled vibrations) signaling. While all Schizocosa males incorporate some form of seismic courtship signaling, a subset also incorporates visual signals (Stratton 2005). Among the visual signaling species, some possess pigmentation or large brushes of hair on their forelegs, which are waved during courtship, while others lack foreleg ornamentation (Stratton 2005; Framenau and Hebets 2007). The observed variation in multimodal courtship signaling among members of the genus allows for comparisons between closely related species and facilitates an understanding of the evolution and function of multimodal signaling in this group.

Using a combination of signal isolation and video playback experiments, recent studies have explored multimodal signal function in two closely related Schizocosa species - S. uetzi and S. stridulans. Males of both species possess ornamentation on their forelegs and produce multimodal courtship displays (seismic plus visual). In order to understand the relative importance of each signaling modality, researchers designed mating arenas that prevented the transmission of each signaling modality independently. For example, to remove the seismic signal, males and females were placed on separate substrata, thereby removing the effective transmission of their substratum-coupled vibrations. Similarly, in order to remove the visual signal, an opaque barrier was placed in between a courting male and female, removing the successful transmission of visual signals. Using such signal isolation techniques, $S$. uetzi females were found to be more receptive to seismic signals as compared to visual signals, while $S$. stridulans females responded equally to signals in both modalities (Hebets and Uetz 2000). These results suggested that the seismic and visual courtship signals are redundant for $S$. stridulans, but nonredundant for $S$. uetzi. However, follow-up experiments examining actual copulation frequency in the presence versus absence of seismic and visual signals demonstrated that for both species, seismic signaling was crucial for mating success, while the visual signal had no impact on mating frequency suggesting nonredundancy (Hebets and Uetz 2000; Hebets 2005, 2008).

Results of video playback experiments add an interesting twist to the story. For both $S$. uetzi and S. stridulans, courtship sequences were digitized and modified into three separate video loops in which the male foreleg ornamentation was modified in the following way: (1) brushes of hair were added (S. uetzi) or enlarged (S. stridulans) on the male's foreleg tibiae- "brushes" video, (2) no changes were made to the male - "control" video, and (3) all pigmentation and brushes were re- 


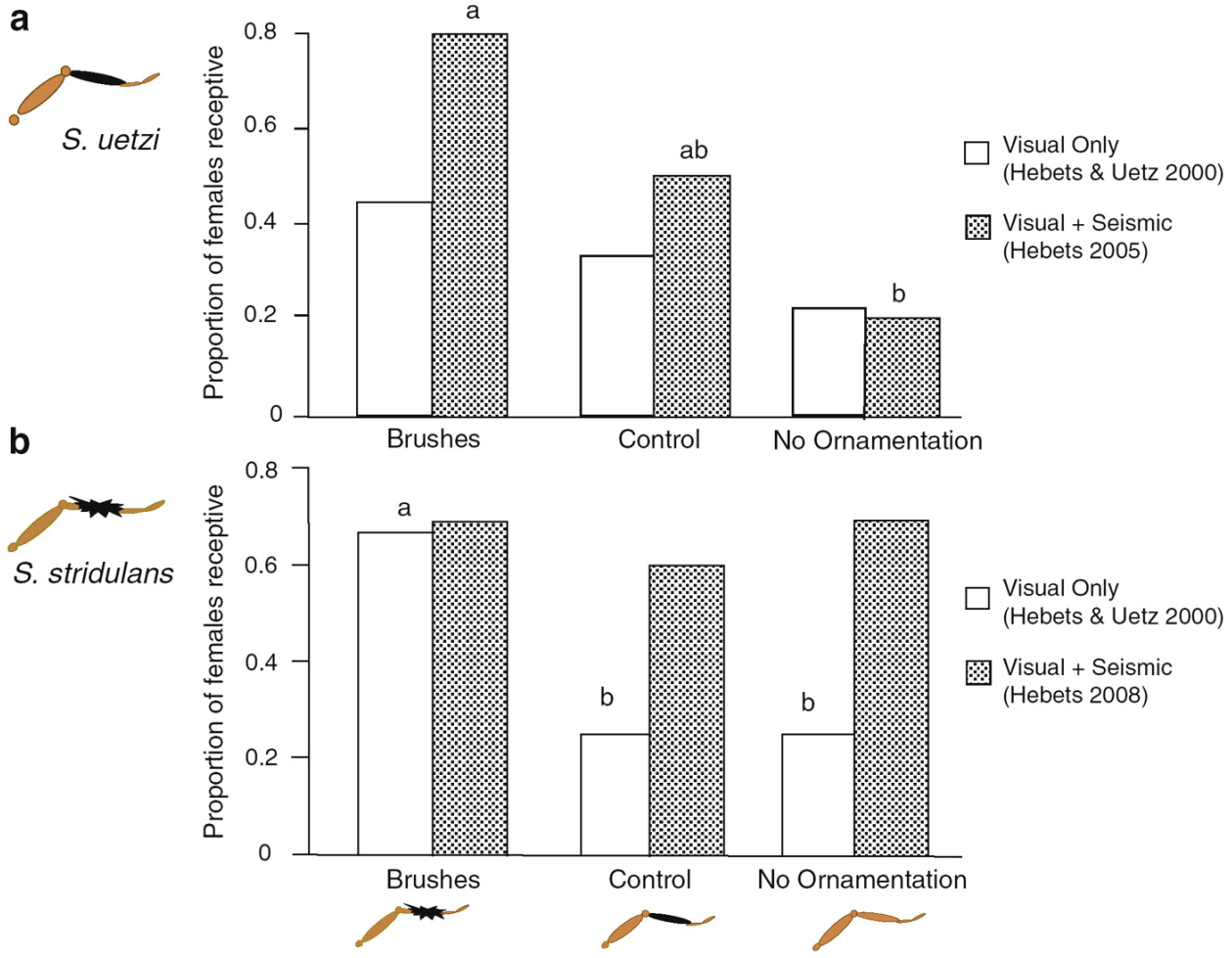

Figure 2. Compilation figure (using data from Hebets 2005, 2008; Hebets and Uetz 2000) depicting female receptivity responses to video playbacks of courting conspecific males in the presence (black bars) and absence (white bars) of a seismic courtship signal for (a) Schizocosa uetzi and (b) S. stridulans.

moved from the foreleg tibiae - "no ornamentation" video. Conspecific female receptivity was then assessed to playbacks of these courtship sequences both in the presence and absence of a seismic courtship signal. When the seismic signal was absent, S. uetzi females did not distinguish among the visual courtship sequences. However, in the presence of a seismic courtship signal, females were more likely to display receptivity to the "brushes" video versus the "no-ornamentation" video (Hebets 2005; Figure 2a). These results, in combination with others, suggest that the seismic signal of $S$. uetzi functions to alter a female's visual attention (Hebets 2005). In contrast, results from $S$. stridulans show an opposite pattern. In the $a b$ sence of the seismic signal, $S$. stridulans females are more likely to display receptivity to more ornamented males (i.e., "brushes" video). However, in the presence of the seismic signal, female receptivity is independent of the visual stimulus (Hebets 2008) (Figure 2b). The results from $S$. stridulans suggest that the seismic signal is dominant to the visual courtship signal (Hebets 2008). Thus, in two closely related species (see Stratton 2005; Hebets and Vink 2007), multimodal courtship signaling appears to function in very different ways: in $S$. uetzi, the signals interact such that 
the seismic signal appears to alter a female's visual attention, and in S. stridulans, the seismic signal appears dominant to the visual signal.

The above examples were chosen to demonstrate several things: (1) studying multimodal signaling can be extremely complicated and often requires multiple approaches and techniques, (2) multimodal signal function may vary greatly even among closely related species, (3) taxonomic groups for which manipulations of both the signaling environment and the signals themselves are feasible make ideal organisms for studying multimodal signaling, and (4) the comparative approach can be extremely valuable in understanding the evolution of multimodal signaling. We highlight these points here because we feel that these same points are relevant and applicable to crustaceans. Crustaceans not only encompass tremendous diversity in terms of communication systems, lifestyles, and habitat use (to name a few), but they are also extremely tractable, making them an excellent taxonomic group for comparative work on multimodal signal form and function.

\subsection{Example II: Sensory Systems Lend Insight into Multimodal Sig- naling in Squirrels}

Before finally turning our focus to crustaceans, we wish to provide another brief example of multimodal signaling, this time borrowing from the vertebrate literature. We chose this example because it highlights the importance of natural history information and knowledge of an organism's basic anatomy and physiology - knowledge that appears to be abundant among crustacean biologists. Specifically, our example illustrates how selection for sensory integration in a foraging context has potentially facilitated the evolution of multimodal signaling in a communication context. Our example comes from interactions between California ground squirrels, Spermophilus beecheyi, and their rattlesnake predators (Crotalus oreganus).

Although rattlesnakes are sensitive to visual stimuli at close range, they also possess pit organs, a highly specialized sensory system that enables them to detect infrared radiation or "radiated heat" at a distance. Bimodal neurons in the rattlesnake's optic tectum respond to both infrared and visual stimuli (Hartline et al. 1978; Newman and Hartline 1981), creating a visual and thermal representation of the snake's environment and enabling the integration of visual and thermal stimuli. These rattlesnakes will often feed on pups of California ground squirrels. In defense of their vulnerable young, adult ground squirrels vigorously confront predatory snakes with a suit of antipredator behaviors, including a visual signal called tail flagging in which they wave their piloerected tails from side-to-side (Owings and Coss 1977). Recently, a closer examination of this tail flagging display revealed that when the signal is directed towards a rattlesnake, the squirrels increase the temperature of their tails by $2-3{ }^{\circ} \mathrm{C}$ (Figure $3 \mathrm{a}$ ). Interestingly, the squirrels do not increase their tail temperature when tail flagging to infrared insensitive gopher snakes, Pituophis melanoleucus, (Figure 3b; Rundus et al. 2007). Thus, when encountering an infrared sensitive snake, California 


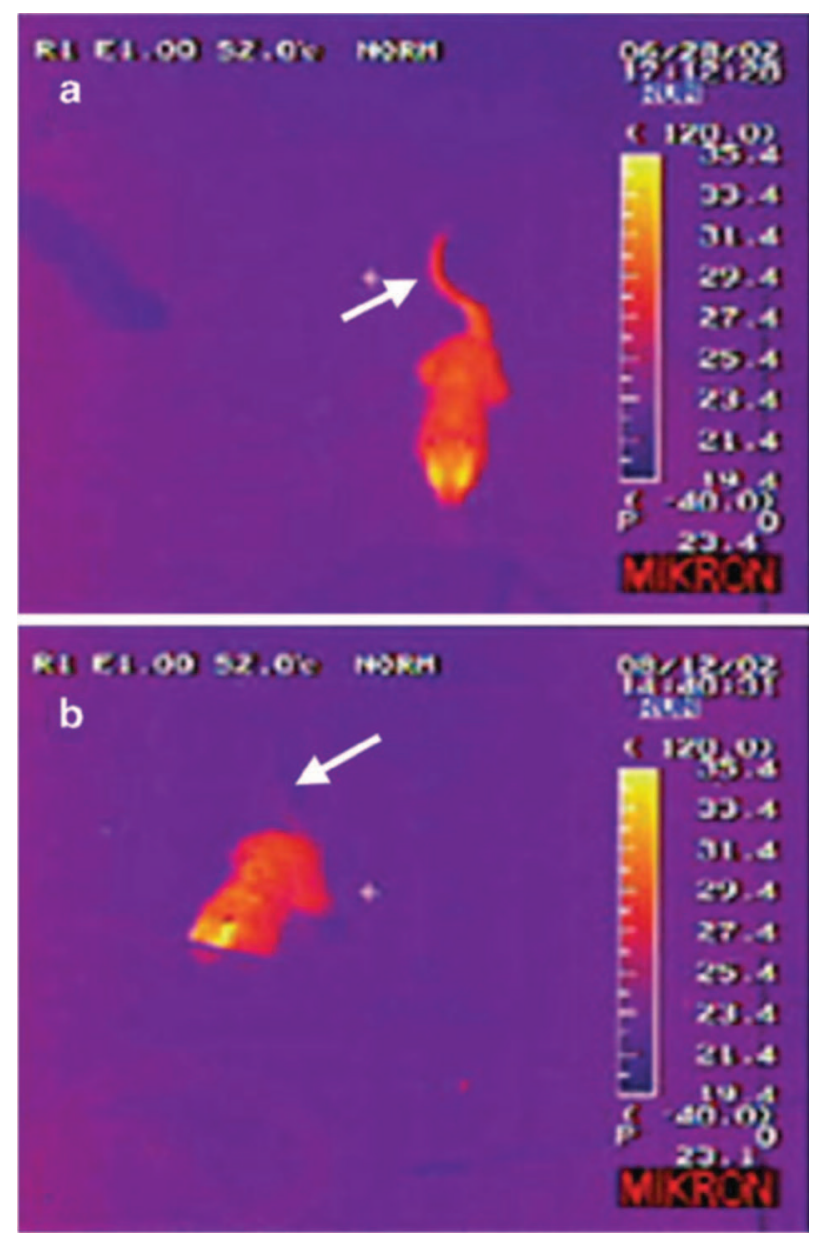

Figure 3. Infrared video frames of a squirrel interacting with (a) a rattlesnake and (b) a gopher snake during experimental trials. Lighter pixel color corresponds to warmer object surface temperature. Note that the tail regions of the squirrel, referenced by the white arrows, are considerably warmer than background during the rattlesnake trial, but not the gopher snake trial. Figure adapted from Rundus et al. (2007).

ground squirrels produce a multimodal (thermal and visual) signal, which capitalizes on the multimodal sensory specializations of their signal target. The addition of the infrared component to the visual tail flagging shifts rattlesnakes from predatory to defensive behavior to a greater degree than the visual component alone (Rundus et al. 2007), suggesting an enhancing effect of this additional component. Again, we highlight this example because it clearly demonstrates how an understanding of an animal's sensory system might help direct our studies of communication, enabling the discovery of signals or components that may lie beyond our human perceptual capabilities. Given the wealth of knowledge regarding crustacean sensory systems, a sensory systems approach might prove valuable for understanding the evolution of crustacean multimodal signaling. 


\section{Chemical Communication and Multimodal Signaling}

Chemical signaling is considered to be the oldest and most widespread channel for communication (Bradbury and Vehrencamp 1998; Johansson and Jones 2007). Not surprisingly then, examples of animals that incorporate chemical signals into multimodal displays are numerous (e.g., $>50 \%$ of provided multimodal signaling examples in a recent review involve a chemical component: see Tables 2 and A1 in Partan and Marler 2005). Interestingly, of those reviewed studies that included chemical signals and examined the redundancy of signal components, $>60 \%$ of the taxa used were invertebrates (Partan and Marler 2005). We point this out here to stress the value of invertebrate taxa, such as crustaceans, in advancing our understanding of multimodal signaling.

Despite the prevalence of chemical components in multimodal displays, many of the theoretical and functional studies of multimodal signaling involve audiovisual displays (see Candolin 2003; Hebets and Papaj 2005). This likely reflects a greater difficulty in identifying and characterizing chemical signals as well as an effect of observer bias. For example, relating a receiver's response to the presence/absence of a chemical stimulus is relatively straightforward, yet it is far less intuitive to correlate variation in a chemical stimulus to variation in signaler attributes. Studying multimodal signals that incorporate a chemical component can face an additional challenge-discontinuity of components in space and time. A majority of studies of multimodal signal function have focused on signal components that are closely linked in either space (e.g., infrared and thermal, visual and mechanical) or time (e.g., visual and acoustic). Given the wide area across which chemical signals often disperse and the often extended time frames for their emission, interpreting relationships between components, particularly intersignal interactions, can become quite challenging. In fact, as mentioned earlier, little is known about the role of synchrony of multimodal signal components (but see Narins et al. 2005) and complex displays that contain "immediate" or "fast acting" components (e.g., visual, acoustic) paired with more "tonic" components (chemical) would be ideal candidates for further exploration.

\section{Crustaceans and Multimodal Signaling}

\subsection{Chemical and Hydrodynamic Multimodal Signaling}

The sensory system of any animal has presumably evolved under sources of selection generated by its environment. In an aquatic environment, for example, one expects animals to be able to sense fluid dynamics, since chemical cues important for survival are necessarily embedded in these movements (Mellon 2007). Not surprisingly then, aquatic crustaceans possess multiple fluid-flow detectors, chemoreceptors, and even bimodal chemo-mechanoreceptors (Hallberg and Skog, Chap. 6). A wealth of literature exists on all of these sensory structures and the bimodal chemo-mechanosensory sensilla specifically have been examined ex- 
tensively in the spiny lobster Panulirus argus (Cate and Derby 2001, 2002). The widespread existence of these bimodal sensilla is intriguing and the significance of combining these two modalities into a single sensillum has received much attention from neurobiologists (see M. Schmidt and D. Mellon, "Neuronal Processing of Chemical Information in Crustaceans," Chap. 7 in Breithaupt \& Thiel, eds., Chemical Communication in Crustaceans, 2011). From a communication perspective, given that individuals are capable of such sensory integration at the peripheral sensory level, one might expect at least some signalers to capitalize on this in a similar way that the California ground squirrels capitalize on the bimodal (visual plus thermal) sensory perception of rattlesnakes ("sensory bias for multisensory integration" hypothesis of Sensory Constraints - Hebets and Papaj 2005).

In addition to the above-highlighted capacity to receive and process bimodal cues (chemical plus hydrodynamic), aquatic crustaceans must often couple chemical signal production with the generation of water currents in order to transmit these signals-making many chemical signals obligately tied to hydrodynamic cues. For example, in lobsters, urine containing chemical signals is "carried by anteriorly directed gill currents" (Atema 1985). The fan organs of crayfish have also been shown to enhance the flow of chemical information (Breithaupt 2001) and forward-directed gill currents were proposed as the means of transporting urine signals during male-male contests in the crayfish Astacus leptodactylus (Breithaupt and Eger 2002). Similar fanning behavior is observed in terrestrial animals as a means to disperse chemical signals (e.g., male sac-winged bats, Saccopteryx bilineata; Voigt and von Helversen 1999). Despite the often obligatory tie between chemical and hydrodynamic cues in many crustaceans, some receivers still focus mostly on a single modality. For example, the mating behavior in copepods seems to fall into two separate strategies - species in which males follow chemical trails left by females, and those for which males perform a tandem hopping behavior, mediated primarily by hydrodynamic cues left by a female (see Yen and Lasley, Chap. 9). Ultimately, in both groups, the final leap of the male (just before capturing the female) is thought to be mediated by hydrodynamic cues (Yen et al. 1998).

Given that (1) receivers can simultaneously detect both chemical and hydrodynamic cues and (2) hydrodynamic cues are often obligately tied to chemical signal transmission, it seems reasonable to expect that in some circumstances, selection has acted on the hydrodynamic cues (i.e., current production) for a communication function. Indeed, hydrodynamic signals are used in agonistic displays of hermit crabs, though here the evidence of their use in a multimodal signal is only via a temporal coupling with a visual display (Barron and Hazlett 1989). Multiple studies using various crustacean groups have demonstrated both the production of several distinct types of water currents during social interactions (Figure 4) and the context-dependent production of such water currents (e.g., Herberholz and Schmitz 2001; Bergman and Moore 2005a; Simon and Moore 2007), suggesting a function in signaling. Some of these studies have confirmed that hydrodynamic currents act in conjunction with chemical signals for multimodal communication (e.g., Bergman and Moore 2005b; Simon and Moore 2007). Despite our knowledge of the existence of these multimodal displays, however, their function remains unknown. For example, while Simon and Moore (2007) suggest that 


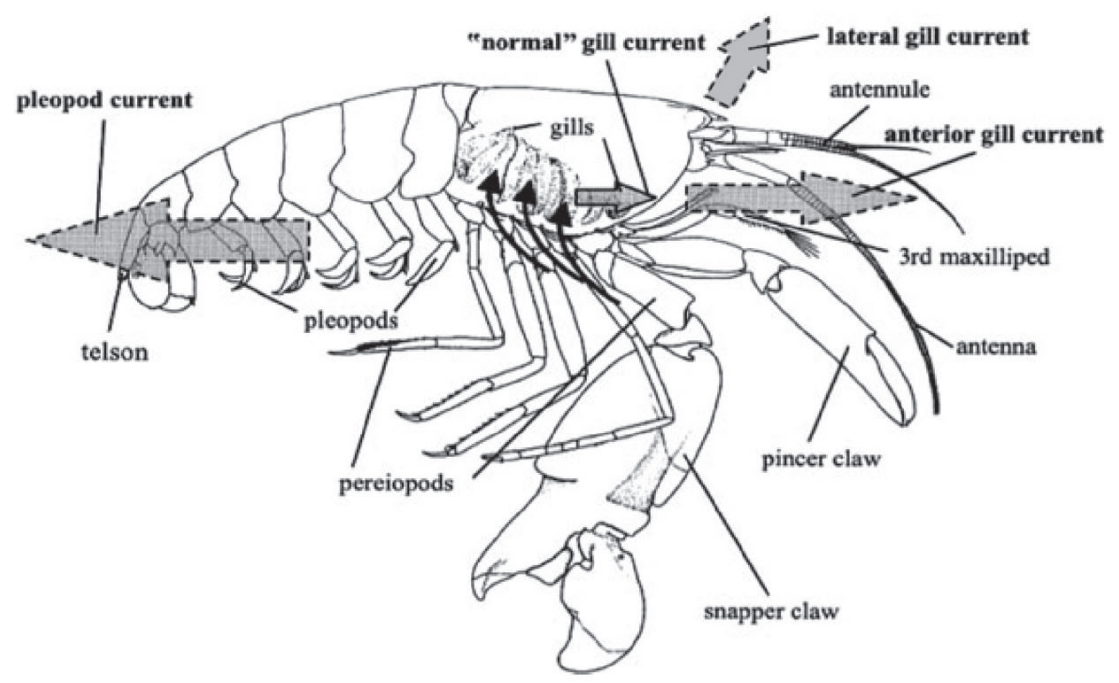

Figure 4 Drawing (lateral view) of a snapping shrimp (originally modified after Kim and Abele 1988) showing four different water currents (gray arrows): the "normal" gill current, the lateral gill current, the anterior gill current, and the pleopod current. Black arrows show the direction of water entering the gill chamber (from Herberholz and Schmitz 2001)

the water currents generated by the crayfish Orconectes rusticus during male-female pairings do not provide information but instead simply facilitate the transmission of the information-carrying chemical signal, this hypothesis has not been explicitly tested. In summary, much work remains regarding understanding the function of combining chemical plus hydrodynamic stimuli, and the prevalence of these combined modalities among crustaceans begs for such future work.

\subsection{Chemical and Visual Multimodal Signaling}

While chemical and hydrodynamic signals may be functionally connected in aquatic environments, the addition of visual signals makes intuitive sense for animals that live in terrestrial or shallow-water environments. Not surprisingly, numerous studies have documented an interaction between chemical and visual cues in crustaceans across a variety of contexts (e.g., foraging, navigation, antipredator behavior, intraspecific interactions, etc.). For example, in a study examining individual recognition, Gherardi and Tiedemann (2004) used a signal isolation design to examine the behavior of the hermit crab, Pagurus longicarpus, towards familiar or unfamiliar conspecifics. They examined individual behavior in the presence of visual only, chemical only, or visual plus chemical cues. Although they found olfaction to be the dominant modality used for individual recognition, the combination of visual and olfactory cues resulted in an enhancement of the receiver's response (Gherardi and Tiedemann 2004; Table 1), sug- 
gesting some benefit to bimodal (chemical plus visual) sensory acquisition. In a similar study examining individual recognition in the crayfish Cherax destructor, Crook et al. (2004) were able to demonstrate that individuals can discriminate familiar from unfamiliar opponents using either chemical only or visual only cues. Their results suggest that chemical and visual cues may act as backups to each other-potentially providing redundant information (Crook et al. 2004). Finally, using similar cue/signal isolation designs, numerous studies have also demonstrated that the presence of an odor influences an animal's orientation response to a visual stimulus, again suggesting an interaction between chemical and visual stimuli (Chiussi et al. 2001; Diaz et al. 2001; Chiussi and Diaz 2002; Huang et al. 2005; Table 1).

Several studies have also examined the importance of chemical and visual stimuli in the context of mate choice. A recent study paired male and female red swamp crayfish, Procambarus clarkii, in the presence of visual only, chemical only, or visual plus chemical cues and examined the influence of bimodal information on mate choice (Aquiloni and Gherardi 2008). The authors found that females chose larger males only in the presence of both stimuli and that, when given only one cue, females often acted aggressively (Aquiloni and Gherardi 2008; Table 1). In contrast, a male's choice of larger females was based solely on chemical cues, but they engaged in mating behavior most when both stimuli were present (Aquiloni and Gherardi 2008). Results of this study suggest that males and females may use bimodal information differently. A second example is provided by Acquistapace et al. (2002) in which the authors demonstrate that male crayfish, Austropotamobius pallipes, react to the combination of chemical and visual cues from conspecific females more than they do to chemical cues in isolation. Although this latter study examined male responsiveness across four treatments (female odor alone, male odor alone, female odor plus visual cues, and male odor plus

Table 1. Evidence for visual and chemical sensory integration in crustaceans

\begin{tabular}{|c|c|c|}
\hline Species & Purpose/context & Reference \\
\hline Uca cumulanta (Fiddler crab) & Predator-avoidance & Chiussi and Diaz (2002) \\
\hline $\begin{array}{l}\text { Synalpheus demani (Snapping } \\
\text { shrimp) }\end{array}$ & Shelter-seeking & Huang et al. (2005) \\
\hline Clibanarius antillensis (Hermit crab) & Shelter-seeking/predator-avoidance & Chiussi et al. (2001) \\
\hline Austropotamobius pallipes (Crayfish) & Sex recognition & $\begin{array}{l}\text { Acquistapace et al. } \\
\qquad(2002)\end{array}$ \\
\hline $\begin{array}{l}\text { Alpheus heterochaelisi (Snapping } \\
\text { shrimp) }\end{array}$ & Mate choice/male competition & Hughes (1996) \\
\hline Callinectes sapidus (Crab) & Mate choice & Kamio et al. $(2008)^{a}$ \\
\hline Procambarus clarkii (Crayfish) & Mate choice & $\begin{array}{l}\text { Aquiloni and Gherardi } \\
\text { (2008) }\end{array}$ \\
\hline Cherax destructor (Crayfish) & Individual recognition & Crook et al. (2004) \\
\hline Pagurus longicarpus (Hermit crab) & Individual recognition & $\begin{array}{l}\text { Gherardi and } \\
\text { Tiedemann (2004) }\end{array}$ \\
\hline
\end{tabular}

a. In addition to visual and chemical signals, the authors also suggest hydrodynamic signals 
visual cues), the authors did not test male responses to visual cues in isolation, making it difficult to interpret their results in terms of multimodal signal function (Acquistapace et al. 2002).

Despite the fact that many of the above-mentioned studies clearly demonstrate an impact of bimodal (visual plus chemical) sensory stimuli on an individual's behavior, they do not necessarily reflect communication and thus may not represent examples of multimodal signaling per se. For example, while the presence versus absence of an individual is clearly a manipulation of visual stimuli, it is unlikely that the individual itself constitutes a signal (i.e., has been selected for a communicative function; see T. Wyatt, "Pheromones and Behavior," Chap. 2 in Breithaupt \& Thiel, eds., Chemical Communication in Crustaceans, 2011). Rather, the presence of an individual might be more appropriately treated as a cue (Bradbury and Vehrencamp 1998). Similarly, the chemical stimuli used in many of the studies may or may not reflect past selection. The distinction between signals and cues is often difficult, especially with respect to complex signaling - where a multimodal display can be a functional unit upon which selection can act, regardless of whether its components are signals or cues (Hebets and Papaj 2005). In many of the above-mentioned cases, further investigation of both chemical and visual stimuli will likely confirm them as true examples of multimodal signaling.

One crustacean species in which chemical plus visual multimodal signaling appears to be important is the snapping shrimp Alpheus heterochaelis. Using signal isolation techniques, Hughes (1996) examined male responses to chemical signals from males and females in isolation and found that male responses were independent of the chemical signal. Next, she presented chemical signals jointly with the visual signal of an open chela display - a visual signal produced by both sexes. She found that the response of the male shrimp to the visual display was dependent on the chemical signal (Hughes 1996). Males responded more to the open chela display in the presence of a male versus female chemical signal (Figures 5 and 6). In addition, the male's response was dependent on the chela size only in the presence of the female chemical signal (Hughes 1996). These experiments clearly show that the chemical signal (male vs. female) alters the male's response to the visual, open chela, display. Although this study preceded many of the theoretical reviews of the topic, it appears to represent an example of multimodal signaling in which one signal provides a context in which a perceiver can interpret and respond to a second (Hebets and Papaj 2005).

Another recent study documents a complex courtship display that combines visual, chemical, and hydrodynamic signals into a trimodal signal. Prior studies established that male blue crabs, Callinectes sapidus, have complex chemical signals, likely incorporating both urine and nonurine sources (Bushmann 1999). During courtship, these males sometimes adopt a stationary paddling display in which they paddle their swimming legs while standing in an elevated position with their chelae open (Kamio et al. 2008). The paddling behavior described above increases the volume of water males pump out of their gill chambers, suggesting a potential hydrodynamic signal (Gleeson 1991). In addition, sexually receptive females have previously been shown to respond to models of male crabs in this stationary posture and the exposed blue coloration on the chelae during 


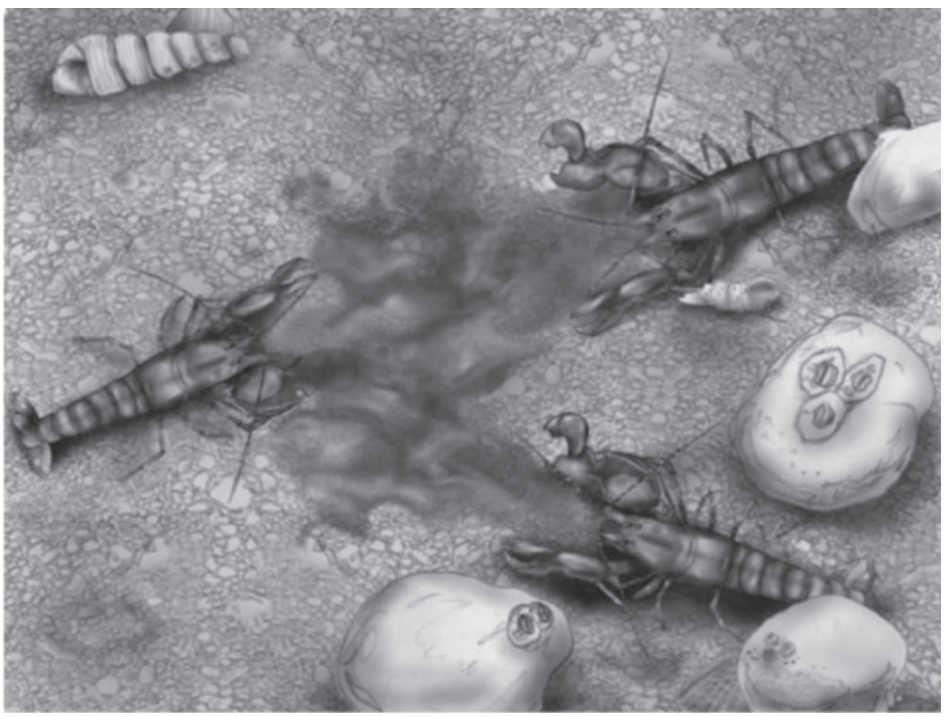

Figure 5. Drawing depicting how male Alpheus heterochaelis (left) respond more to an open chela display in the presence of male (top right) versus female (bottom right) chemical signals. Greater response is artistically represented by male orientation. Drawing by Jorge A. Varela Ramos.

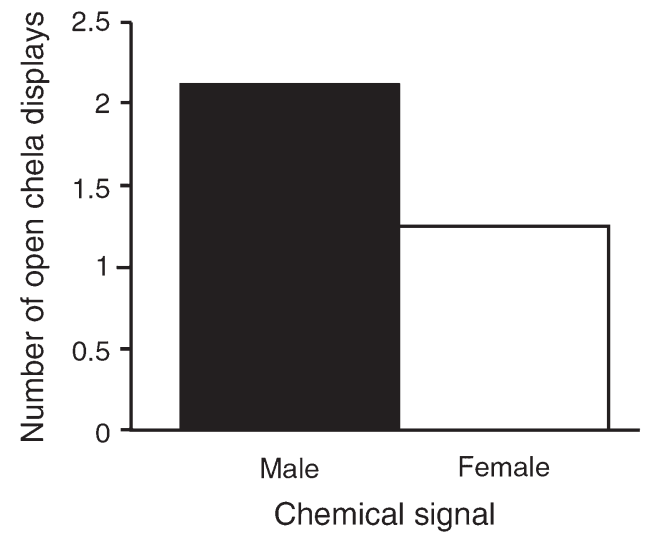

Figure 6. Actual data representing male responses to open chela display (as indicated by the number of open chela displays) in the presence of male versus female chemical signals. Data modified from Hughes (1996), Figure 5.

the spread display suggests a visual signal (Gleeson 1991). Recently, Kamio and colleagues examined the context in which this stationary paddle display was performed. Specifically, they manipulated the male's signaling environment into treatments where females were accessible versus inaccessible (putatively mimicking low visibility environments in which females may be recessed in a refuge) and assessed their use of stationary paddling. Results demonstrated that males were more likely to engage in stationary paddling in environments where females were inaccessible versus accessible (see Figure 7). In addition, the authors were able to verify that a larger volume of water and a larger velocity of flow were generated during the stationary paddling display than when males were not per- 
$\square$ Cradle carry

- Courtship stationary paddling

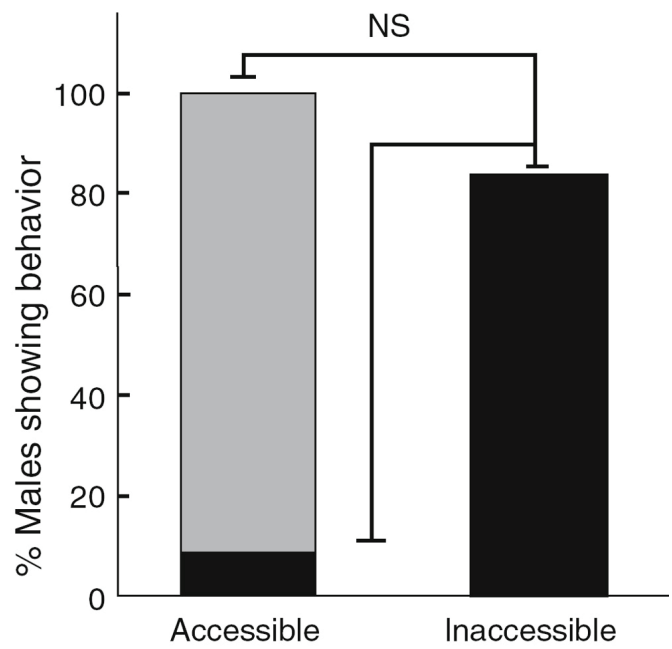

Figure 7. Courtship-related behaviors shown by male blue crabs in the presence of an accessible female and in the presence of an inaccessible female. While there was no significant difference in total courtship behavior (Carry cradle + Courtship paddling) between the two testing conditions, the number of males exhibiting courtship stationary paddling was significantly higher in the inaccessible condition. From Kamio et al. 2008.

forming this display (Kamio et al. 2008). Taken together, the authors suggest that the trimodal aspect of male courtship display is context-dependent and functions as an efficacy-backup in the presence of environmental variability ("Multiple sensory environments" - sensu Candolin 2003; Hebets and Papaj 2005). Few studies have documented context- or environment-dependent use of multimodal signals (for discussions of multimodal signaling dependent on social context see Leger 1993; Partan and Marler 2005) and thus this study adds a tremendous amount to our understanding of complex signal function.

\section{Future Directions}

To date, numerous researchers and numerous studies have thoroughly examined the effects of different sensory stimuli on crustacean behavior; however, many of these studies are difficult to interpret within the framework of multimodal signaling. For example, many of the experiments discussed previously were not designed from a communication or signaling perspective-many of the experiments did not incorporate a full factorial design; many did not allow for potential intersignal interactions; most did not address the distinction between signals and cues; and none examined signal form in relation to signaler quality, identity, etc. Unfortunately, as a result of the different perspectives from which these studies were conducted, we are currently limited in the conclusions that can be drawn regarding multimodal communication in crustaceans. Regardless, the studies do highlight the importance of bimodal, and even trimodal, stimuli on crustacean behavior and suggest that multimodal signaling, specifically chem- 
ical plus hydrodynamic and visual, is likely prevalent among crustaceans. These studies set the stage for well-designed future work couched in a solid conceptual and theoretical communication framework.

In addition to the various design issues briefly discussed above, most crustacean researchers have not yet explicitly tested hypotheses of multimodal signal function (but see Kamio et al. 2008). To date, most studies have simply involved signal isolation designs in which responses of receivers are assessed to isolated cues/signals. However, many hypotheses of multimodal signal function require an examination of correlations between signaler and signal form and/or require further manipulations of either the signal itself or the signaling environment. Elegant manipulations of crustacean signals have already been conducted in other contexts, demonstrating that crustaceans are indeed amenable to the types of experimental approaches and techniques necessary for addressing questions of multimodal signaling function. For example, signal ablation studies have used reversible blindfolding to occlude visual signals (Breithaupt and Eger 2002) and more recently, numerous creative techniques have been used to manipulate visual signals for playback-style studies. Mirrors have been incorporated into studies to magnify claw size in waving fiddler crabs while controlling for waving rate and waving motion (McLain and Pratt 2007). In addition, robotic male crabs have been implemented in a study aimed at exploring female preference for synchrony (Reaney et al. 2008). The incorporation of these new technologies into studies examining multimodal signal function will surely provide exciting results.

Given the diverse environments in which crustaceans live, they also offer a unique opportunity to study the influence of signaling environment on the evolution of multimodal signaling. Specifically, they offer the possibility to compare and contrast the multimodal communication systems of closely related aquatic versus terrestrial organisms. Along these lines, prior comparative studies examining the reproductive behavior of mantis shrimp and fiddler crabs have already suggested several factors hypothesized to have been important in the evolution of their respective sexual signals (Christy and Salmon 1991). In comparing multimodal signaling between aquatic and terrestrial crustaceans, $a$ priori, we might expect a prevalence of chemical plus hydrodynamic or visual signals in aquatic crustaceans while more terrestrial species might pair visual and acoustic signals. Anecdotally, on a recent trip to Costa Rica, I (EAH) was struck by the multimodal displays of a common beach-side fiddler crab. Upon approach, individuals waved their brightly colored chela in the air while simultaneously producing a sound - resulting in a multimodal display (visual + acoustic) that presumably functions to warn off predators. Acoustic communication is well known among different groups of crustaceans (Müller 1989; Popper et al. 2001), and the combination of visual and acoustic signals has already been reported in mating contexts (e.g., singing and dancing in the ghost crab Ocypode platytarsus - (Clayton 2008)). Although our focus in this chapter has been limited to multimodal signals incorporating chemical components, crustaceans likely encompass numerous examples of multimodal signals that combine other sensory modalities. 


\section{Summary and Conclusions}

Despite the plethora of both theoretical and empirical studies emerging on the topic of multimodal signal evolution and function, innumerable questions remained unanswered. For example, what is the importance of simultaneous versus sequential production, transmission, and perception of signals from multiple modalities on multimodal signal function? How important are various selection pressures such as signaling environment (e.g., aquatic vs. terrestrial), information content or signal purpose, receiver sensory systems, etc. on multimodal signal evolution? Why are certain modalities paired together over others? For many of the reasons highlighted throughout this chapter, we believe that crustaceans are ideal organisms to use for filling in these gaps in our knowledge of multimodal signal evolution and function. As such, we hope that this chapter will inspire future work on crustacean multimodality, as we believe that such work will undoubtedly lead to important contributions to our broader understanding of complex signal evolution.

Acknowledgments - We would like to extend a special thanks to both Martin Thiel and Thomas Breithaupt for the invitation to participate in this book (despite the fact that neither of us knew much of anything at the onset about crustaceans). We have both learned a tremendous amount and have thoroughly enjoyed getting to know some of the arachnid's arthropod relatives. We would also like to thank Martin and Thomas for steering us towards relevant literature and for their excellent editorial comments. In addition, we would like to thank John Christy for pointing us in the right direction for acoustical references. We would also like to thank three anonymous reviewers for incredibly insightful and helpful comments. Finally, we would like to thank all of the crustacean researchers for providing stimulating reading.

\section{References}

Acquistapace P, Aquiloni L, Hazlett BA, Gherardi F (2002) Multimodal communication in crayfish: Sex recognition during mate search by male Austropotamobius pallipes. Can J Zool 80:2041-2045

Aquiloni L, Gherardi F (2008) Assessing mate size in the red swamp crayfish Procambarus clarkii: Effects of visual versus chemical stimuli. Freshwater Biol 53:461-469

Atema J (1985) Chemoreception in the sea: Adaptations of chemoreceptors and behaviour to aquatic stimulus conditions. Soc Ex Biol Symp 39:386-423

Barron LC, Hazlett BA (1989) Directed currents: A hydrodynamic display in hermit crabs. Mar Behav Physiol 15:83-87

Bergman DA, Moore PA (2005a) Prolonged exposure to social odours alters subsequent social interactions in crayfish (Orconectes rusticus). Anim Behav 70:311-318

Bergman DA, Moore PA (2005b) The role of chemical signals in the social behavior of crayfish. Chem Senses 30:i305-i306

Bradbury JW, Vehrencamp SL (1998) Principles of animal communication. Sinauer Associates, Massachusetts

Breithaupt T (2001) Fan organs of crayfish enhance chemical information flow. Biol Bull 200:150-154

Breithaupt T, Eger P (2002) Urine makes the difference: Chemical communication in fighting crayfish made visible. J Exp Biol 205:1221-1231 
Bushmann PJ (1999) Concurrent signals and behavioral plasticity in blue crab (Callinectes sapiduss> Rathbun) courtship. Biol Bull 197:63-71

Candolin U (2003) The use of multiple cues in mate choice. Biol Rev 78:575-595

Cate HS, Derby CD (2001) Morphology and distribution of setae on the antennules of the Caribbean spiny lobster Panulirus argus reveal new types of bimodal chemo-mechanosensilla. Cell Tiss Res 304:439-454

Cate HS, Derby CD (2002) Hooded sensilla homologues: Structural variations of a widely distributed bimodal chemomechanosensillum. J Comp Neurol 444:345-357

Chiussi R, Diaz H (2002) Orientation of the fiddler crab, Uca cumulanta: Responses to chemical and visual cues. J Chem Ecol 28:1787-1796

Chiussi R, Diaz H, Rittschof D, Forward RB (2001) Orientation of the hermit crab Clibanarius antillensis: Effects of visual and chemical cues. J Crust Biol 21:593-605

Christy JH, Salmon M (1991) Comparative-studies of reproductive-behavior in mantis shrimps and fiddler-crabs. Am Zool 31:329-337

Clayton D (2008) Singing and dancing in the ghost crab Ocypode platytarsus (Crustacea, Decapoda, Ocypodidae). J Nat Hist 42:141-155

Crook R, Patullo BW, MacMillan DL (2004) Multimodal individual recognition in the crayfish Cherax destructor. Mar Freshwater Behav Physiol 37:271-285

Diaz H, Orihuela B, Forward RB, Rittschof D (2001) Effects of chemical cues on visual orientation of juvenile blue crabs, Callinectes sapidus (Rathbun). J Exp Mar Biol Ecol 266:1-15

Framenau VW, Hebets EA (2007) A review of leg ornamentation in male wolf spiders, with the description of a new species from Australia, Artoria schizocoides (Araneae, Lycosidae). J Arachnol 35:89-101

Gherardi F, Tiedemann J (2004) Chemical cues and binary individual recognition in the hermit crab Pagurus longicarpus. J Zool 263:23-29

Gleeson RA (1991) Intrinsic factors mediating pheromone communication in the blue crab, Callinectes sapidus. In: Martin JW, Bauer RT (eds) Crustacean sexual biology. Columbia University Press, New York

Guilford T, Dawkins MS (1991) Receiver Psychology and the Evolution of Animal Signals. Anim Behav 42:1-14

Hartline PH, Kass L, Loop MS (1978) Merging of modalities in the optic tectum: Infrared and visual integration in rattlesnakes. Science 199:1225-1229

Hazlett BA, McLay C (2000) Contingencies in the behaviour of the crab Heterozius rotundifrons. Anim Behav 59:965-974

Hebets EA (2005) Attention-altering interaction in the multimodal courtship display of the wolf spider Schizocosa uetzi. Behav Ecol 16:75-82

Hebets EA (2008) Seismic signal dominance in the multimodal courtship display of the wolf spider Schizocosa stridulans Stratton 1991. Behav Ecol 19:1250-1257

Hebets EA, Papaj DR (2005) Complex signal function: Developing a framework of testable hypotheses. Behav Ecol Sociobiol 57:197-214

Hebets EA, Uetz GW (2000) Female responses to isolated signals from multimodal male courtship displays in the wolf spider genus Schizocosa (Araneae: Lycosidae). Anim Behav 57:865-872

Hebets EA, Vink CJ (2007) Experience leads to preference: Experienced females prefer brushlegged males in a population of syntopic wolf spiders. Behav Ecol 18:1010-1020

Herberholz J, Schmitz B (2001) Signaling via water currents in behavioral interactions of snapping shrimp (Alpheus heterochaelis). Biol Bull 201:6-16

Huang D, Rittschof D, Jeng M (2005) Visual orientation of the symbiotic snapping shrimp Synalpheus demani. J Exp Mar Biol Ecol 326:56-66 
Hughes M (1996) The function of concurrent signals: Visual and chemical communication in snapping shrimp. Anim Behav 52:247-257

Johansson BG, Jones TM (2007) The role of chemical communication in mate choice. Biol Rev 82:265-289

Johnstone RA (1996) Multiple displays in animal communication: 'Backup signals' and 'multiple messages'. Phil Trans Roy Soc Lond Ser B 351:329-338

Kamio M, Reidenbach MA, Derby CD (2008) To paddle or not: Context dependent courtship display by male blue crabs, Callinectes sapidus. J Exp Biol 211:1243-1248

Leger DW (1993) Contextual sources of information and responses to animal communication signals. Psychol Bull 113:295-304

McLain DK, Pratt AE (2007) Approach of females to magnified reflections indicates that claw size of waving fiddler crabs correlates with signaling effectivess. J Exp Mar Biol Ecol 343:227-238

Mellon D (2007) Combining dissimilar senses: Central processing of hydrodynamic and chemosensory inputs in aquatic crustaceans. Biol Bull 213:1-11

Møller AP, Pomiankowski A (1993) Why have birds got multiple sexual ornaments. Behav Ecol Sociobiol 32:167-176

Müller W (1989) Untersuchungen zur akustisch-vibratorischen Kommunikation und Ökologie tropischer und subtropischer Winkerkrabben. Zool Jb Abt Syst Ökol Geogr Tiere 116:47-114

Narins PM, Grabul DS, Soma KK, Gaucher P, Hodl W (2005) Cross-modal integration in a dartpoison frog. Proc Nat Acad Sci USA 102:2425-2429

Newman EA, Hartline PH (1981) Integration of visual and infrared information in bimodal neurons of the rattlesnake optic tectum. Science 213:789-791

Owings DH, Coss RG (1977) Snake mobbing by California ground squirrels: Adaptive variation and ontogeny. Behavior 62:50-69

Partan S, Marler P (1999) Behavior - Communication goes multimodal. Science 283:1272-1273

Partan SR, Marler P (2005) Issues in the classification of multimodal communication signals. Am Nat 166:231-245

Popper AN, Salmon M, Horch KW (2001) Acoustic detection and communication by decapod crustaceans. J Comp Phys A 187:83-89

Reaney LT, Sims RA, Sims SWM, Jennions MD, Backwell PRY (2008) Experiments with robots explain synchronized courtship in fiddler crabs. Curr Biol 18:R62-R63

Rowe C (1999) Receiver psychology and the evolution of multicomponent signals. Anim Behav 58:921-931

Rundus AS, Owings DH, Joshi SS, Chinn E, Giannini N (2007) Ground squirrels use an infrared signal to deter rattlesnake predation. Proc Nat Acad Sci USA 104:14372-14376

Simon JL, Moore PA (2007) Male-female communication in the crayfish Orconectes rusticus: The use of urinary signals in reproductive and non-reproductive pairings. Ethology 113:740-754

Stratton GE (2005) Evolution of ornamentation and courtship behavior in Schizocosa: Insights from a phylogeny based on morphology (Araneae, Lycosidae). J Arachnol 33:347-376

Uetz GW, Roberts JA (2002) Multisensory cues and multimodal communication in spiders: Insights from video/audio playback studies. Brain Behav Evol 59:222-230

Voigt CC, von Helversen O (1999) Storage and display of odour by male Saccopteryx bilineata (Chiroptera, Emballonuridae). Behav Ecol Sociobiol 47:29-40

Yen J, Weissburg MJ, Doall MH (1998) The fluid physics of signal perception by mate-tracking copepods. Phil Trans Roy Soc Ser B 353:787-804 